\title{
Polysomnographic Correlates for the Risk of Relapse in Detoxified Opiate-Misuse Patients
}

This article was published in the following Dove Press journal:

Neuropsychiatric Disease and Treatment

\author{
Ahmed Rady (iD' \\ Jaidaa Mekky ${ }^{2}$ \\ Tarek Moulokheya' \\ Ahmed Elsheshai ${ }^{3}$ \\ 'Department of Psychiatry, Alexandria \\ University School of Medicine, \\ Alexandria, Egypt; ${ }^{2}$ Department of \\ Neurology, Alexandria University School \\ of Medicine, Alexandria, Egypt; ${ }^{3} \mathrm{El}$ \\ Mamoura Psychiatric Hospital, \\ Alexandria, Egypt
}

Introduction: Substance abuse is a complex issue requiring multi-disciplinary management. Protracted abstinence syndrome leads to persistent discomfort even after detoxification lasting months, resulting in poor sleep duration and quality. Despite being a major contributor to relapse and sleep disturbances, no objective assessment procedures to monitor sleep after the detoxification have been reported. The study aims to assess sleep structure after detoxification via polysomnography and psychometrically assess sleep over the next 6 months in abstinent and relapsing patients.

Methods: Sixty successfully detoxified males $(35.8 \pm 7.3$ years $)$ with a history of heroin abuse (DSM-V) (mean composite scores (ASI): $1.3 \pm 0.06,3.48 \pm 0.38,5.45 \pm 0.63,1.2 \pm$ $0.19,3.93 \pm 0.9$, and $2.61 \pm 0.8$ for medical status, employment/support status, alcohol/drugs status, legal status, family/social relationships, and psychiatric status, respectively) were recruited immediately following their detoxification, without giving additional psychoactive substances or medications. Polysomnography was done in the second week following detoxification to allow washout of medications, followed by a monthly sleep assessment through sleep diary and daytime sleepiness using a visual analog scale. Relapse was proved by a urine test. Polysomnographic parameters and ASI subscales for relapsing and nonrelapsing participants were compared using a 2-tailed Student's $t$-test $(\mathrm{p}<0.05)$.

Results: Eighteen participants relapsed by 6 months (12 by 3 months). Only the ASI legal problems score was significantly different for the two groups $(\mathrm{p}=0.001)$. The differences were significant for NREM stages I $(\mathrm{p}=0.001)$ and II $(\mathrm{p}=0.002)$, bilateral limb movement $(\mathrm{p}=0.009)$, and arousal indices $(\mathrm{p}=0.001)$.

Conclusion: Measuring polysomnographic parameters (percentage of NREM I and II, arousal index, and limb movement index) for sleep disturbance in detoxified heroin-abuse patients can be potential predictors for relapse in a 6-month follow-up.

Keywords: protracted abstinence, heroin, opiate, polysomnography, relapse

\section{Introduction}

The adolescent population in Egypt has increased in size by $10 \%$, from $20 \%$ in the 1980 s to become $30 \%$ of the population in $2006 .^{1}$ Fiscal development brought about a reorganization of the societal structure in terms of wealth distribution leading to a change in lifestyle and hence, more lifestyle diseases. An enormous rise in the number of people dealing with addiction problems and the increasing admissions in psychiatric hospitals and public institutions, along with rising numbers of registered residential private facilities as addiction centers and follow-up care units are suggestive of this trend. ${ }^{2,3}$ In one of the initial surveys in 1996 for Egypt's National Addiction Program, fairly low (6\%) lifetime drug use rates were
Correspondence: Ahmed Rady Alexandria University School of Medicine, 29 Nabi Daniel St., Alexandria 21/31,

Egypt

Tel +2 01282441053

Email ahmed.rady@alexmed.edu.eg 
reported. ${ }^{4}$ Ever since, several other endeavors at recording substance abuse in Egypt have been made, ${ }^{5}$ but no such data for lifetime prevalence rates are available. ${ }^{6}$ The literature contains data collected from surveying special population subgroups like students, limiting the scope of generalizing the results to older groups. ${ }^{7}$

In 2013, a community survey studying the prevalence of alcohol and substance use in 8 Egyptian governorates $(\mathrm{N}=40,083)$ was conducted which reported a $9.6 \%$ lifetime prevalence of using any addictive substance, and a prevalence of $1.6 \%$ of abuse/dependence in the study sample. ${ }^{8}$ The use of drugs for occasional or recreational purposes was at a lower rate (3.3\%) as against $4.64 \%$ for regular drug use in the sample studied. A similar result was obtained while studying current drug use (1-month prevalence).

As described in DSM-5, addiction is a complex multifaceted disorder with major components as over-implication in behaviors at risk, development of physiological and psychological withdrawal symptoms when abruptly stopped, as well as tolerance manifesting as the need to progressively increase the dose to attain the same effect. ${ }^{9}$ About $80 \%$ of users with a drug-use disorder relapse even after maintaining fairly long periods of abstinence or sobriety, known as the Protracted Withdrawal/Protracted Abstinence (PA) phase. This chronicity of the disease coupled with a higher incidence of backsliding poses difficulty in achieving effective treatment. As observed in clinical settings, symptoms of the PA phase last longer, usually beyond the acute detoxification and withdrawal periods. It is often proposed that treating the PA phase may offer a new potential approach to prevent relapses. However, since the clinical presentation of a patient suffering from the PA phase may not be a complete contrast to the intoxicated state, and may still retain some effects from the drug use besides revealing some pre-existing co-morbidities, the use of this term is still controversial. ${ }^{10}$

Earlier, PA was explained as the state of psychological unrest following a period (initial months) of abstinence from heroin use. Lately, other domains such as cognitive, affective, and behavioral have also been found to be disturbed in patients in the PA phase. ${ }^{10}$ The involvement of the affective component presents as bouts of depression, anxiety, inner tension, irritability, nervousness, and feelings of discomfort and dullness without any precipitating cause, often resulting in heroin craving and sleep disturbance. Thinking about past drug use and drug paraphernalia, illusions, dreams, and memories of previous drug experiences form the cognitive component of the disease. Finally, these feelings influence the behavioral element of PA as the need to procure heroin by any means. ${ }^{10,11}$ It is often seen in patient-exchanges during their admission time for treatment, patients involve in dialogues about their drug use experiences as a part of their behavioral affection.

To understand the mechanisms of how opioid dependence manifests physiologically, a review from the neurobiological perspective is essential. The current literature on the neurophysiological effects of opioids reports that opioid peptides modulate sensory experiences, induce analgesia, and often influence the onset and continuation of sleep, leading to diminution of arousal and wakefulness. ${ }^{12}$ The Enkephalin-containing neurons are extensively distributed throughout the brain, especially in areas responsible for the slow-wave sleep (SWS) like the solitary tract nucleus, the preoptic area, and the raphe, co-existing with the serotonin receptors. ${ }^{12}$ A localized provision of opioids via the Enkephalin-containing fibers inhibits the locus coeruleus noradrenergic neurons resulting in a state of reduced awakening and increased SWS. ${ }^{12}$

The cleavage of a larger peptide Pro-opiomelanocortin (POMC) leads to the formation of neuropeptides like the adrenocorticotropic hormone (ACTH), $\beta$-lipotropin, and the peptide hormone melanocyte-stimulating hormone (MSH). Further cleavage of $\beta$-lipotropin forms $\beta$ endorphin. ACTH is responsible for maintaining steroid production to counter stressful conditions, while $\mathrm{MSH}$ stimulates the melanin production known to induce sleep and improve SWS. Therefore, the hormones related to stress and sleep as well as the endogenous opioid neuropeptides are closely associated in terms of chemical precursors. ${ }^{13}$

However, the underlying mechanism to explain how the opioid system affects sleep remains unclear. A proposed mechanism is via the combination of opioids and the neuropeptide vasopressin. This complex influences the sleep state induction and maintenance by affecting the varying circadian mechanism, which is modulated via the suprachiasmatic nuclei (SCN). ${ }^{14,15}$ Vasopressin stimulates endorphin release in the cerebrospinal fluid, whereas opioids promote the pituitary release of vasopressin. ${ }^{16}$ Also, the supra-optic and periventricular nuclei contain both vasopressin and the opioid peptide dynorphin, the concentration of which varies with the circadian cycle. ${ }^{17}$ Hence, it can be assumed that vasopressin and opioids 
function as a part of the neurochemical mechanism controlling the sleep-wake cycle. ${ }^{14,17}$

While the available literature extensively reports on the phenomenon of protracted abstinence in various druguse disorders, hitherto, only a few studies have explored sleep mechanisms and the effect of opioids on sleep in adult humans, despite a well-acknowledged large-scale opioid use and the influence of endogenous opioids on sleep. ${ }^{18}$ Acute and chronic use of $\mu$-opioids has been shown to affect sleep architecture in animals. ${ }^{19}$ Although $\mu$-opioids are known to cause respiratory depression, more so during sleep, respiratory effects, or disorderedbreathing during sleep and thus an altered sleep-structure caused by using $\mu$-opioids has not been studied thoroughly. ${ }^{18,19}$ Therefore, the present study attempts to address the neurophysiological effects of heroin-use disorder on sleep following detoxification, ie, during the protracted abstinence phase. This prospective study assesses polysomnographic parameters and sleepdisturbances as potential predictors of future relapse in opioid-misuse patients.

\section{Methods}

It is a cohort study conducted using an observational study design. The study was approved by the research review board of the Alexandria University School of Medicine, Egypt. Sixty males with heroin substance-use disorder (diagnosed according to the Diagnostic and Statistical Manual of Mental disorders-fifth edition (DSM-V)), were randomly selected and recruited over 3 months (September 2019 to December 2019) from the El Mamoura Psychiatric Hospital, Egypt after successful detoxification (using a standardized alpha-2 agonist assisted protocol), as confirmed by a negative urine screening test and negative naloxone challenge test. Besides the corroboration of detoxification, participants were included if they confirmed no concurrent misuse of other psychoactive substances apart from smoking (which was present in all the participants). Participants were excluded if they were above 50 years of age (to avoid the effect of other medications for chronic illnesses), had severe debilitating general medical conditions, and those with dual diagnoses as evident from the Structured Clinical Interview for Psychiatric Disorders (SCID-I). A written signed consent, strictly abiding by the Declaration of Helsinki, was obtained from all the participants.

\section{Procedure}

A thorough physical and mental state assessment, including the demographic details and the addiction severity index (ASI) scoring, was done for all the participants. A urine screening was done to confirm abstinence.

\section{Alpha-2 Agonist Assisted Detoxification}

Patients underwent detoxification over a period of 14 days where alpha-2 agonist medication was used as the mainstay to control withdrawal symptoms due to increased sympathetic tone. The alpha- 2 agonist, Clonidine, was administered raising the dose gradually over a couple of days (to avoid build-up) to $900 \mu \mathrm{gm} / \mathrm{d}$ for 10 days and then tapered to stop over the last two days. Other supporting medications, namely mirtazapine, gabapentin, paracetamol, and memantine, were used for symptomatic relief as per requirement.

\section{Polysomnography (PSG)}

It was carried out for all recruited participants 1 week after successful detoxification. No medication of any type, except acetaminophen, was allowed during this period. For the week before the sleep study, alcohol use was prohibited and caffeine was restricted to 10-oz.(one beverage or other consumable containing caffeine) before 23:00 hours. All subjects filled a detailed sleep log daily for the 5 nights before the sleep study and throughout the follow-up describing their sleep experience. During these 5 nights and continuing throughout the sleep evaluation, fixed bedtimes and rise times were established for each subject (at least 7 hours per night) to optimize sleep sufficiency. If the sleep log for any subject revealed variations in the average bedtime and rise times (exceeding 1 hour) during the pre-laboratory schedule, the subject was excluded from the study. The PSG recording was carried out in the Sleep Study Unit of the Department of Psychiatry, Alexandria University using the Cadwell 2.2. The electrode montage included electroencephalograms (EEG) recorded from $\mathrm{C} 3$ and $\mathrm{C} 4$, right and left electrooculograms (EOGs) with contralateral mastoid reference, and bipolar chin-cheek electromyography (EMG). Sleep stages were scored in half-minute epochs in strict accordance with the standard sleep scoring criteria of the American Academy of Sleep Medicine (AASM) manual of 2018. ${ }^{20}$ Several sleep architectural measures were derived from the stage-score data. The onset of persistent sleep (sleep latency) was defined as the time of the initial half-minute of sleep leading the first 10 consecutive 
minutes of any non-REM (NREM) sleep recording, including stage 1 sleep, containing no more than 2 minutes of awake or movement time, or the time of the first REM-sleep epoch, whichever is sooner. REM latency was equal to the number of minutes, including waking, from persistent sleep onset to the first appearance of the REM stage. REM density is an index of phasic eye movement activity in REM sleep for each epoch and averaged per minute of REM. The total sleep period (TSP) was defined as the minutes of sleep time from persistent sleep onset to the morning awakening. Sleep efficiency was defined as the TSP divided by the total time in bed from lights out to lights on (TIB). Additionally, the amount of each REM and NREM sleep stage, and awake and movement times were computed as a percentage of TSP. Records were scored in strict accordance with the accepted sleep scoring conventions by sleep technicians who were blinded to the clinical status of the subject. The nightly REM latencies were consensus-scored by all laboratory technicians (4-6) whose overall sleep-scoring reliability exceeded $90 \%$ for all stages.

\section{Relapse Assessment}

A monthly appointment was made with the participants for assessing their tendency for relapse. A subjective sleep assessment using a sleep diary maintained and filled daily by the patient, and an evaluation of daytimesleepiness using a visual analog scale (VAS) was performed. Participants were asked to rate their daytime sleepiness every day by placing a mark on a $10 \mathrm{~cm}$ line, measuring from "very sleepy" to "very alert". 21,22 Distance of the mark as measured from the left end gives a measure of the participant's response. VAS allows the subject to provide a continuum of response, rather than restricting to a single numerical value. ${ }^{23}$ This ensures more sensitivity of the response while reporting a subjective construct like sleepiness by the user, especially while comparing sleepiness as a "within-subject" factor than a "between-subject" for levels that indicate serious pathology. Additionally, it is a brief process, as compared to the mood adjective checklists. ${ }^{23}$

Another measure assessed from the daily logs of the sleep diary maintained by the patient was initial insomnia, described as the subjective feeling of difficulty to get into sleep as reported by participants. The number of days of a month for which the subject experienced initial insomnia was documented for the next 6 months.

\section{Addiction Severity Index (ASI) Scale}

This scale was used as an assessment parameter descriptive of the user's addiction characteristics, and the total, as well as various sub-groups, were compared. It measures the impact of the behavioral component of addiction on the user's life. Seven areas, namely medical problems, employment/financial aspects, drug problems, problems with alcohol use, illegal activity was undertaken while intoxicated, problems with family and social relations, and psychological effects, are scored based on lifetime use and use in the past 30 days. A higher total score is suggestive of the extent of the influence addiction can have on a person's societal conduct. The various components of the scale cover the various aspects, which if deranged require great attention and intensive treatment. However, there are conflicting views regarding the reliability of the ASI as a meaningful predictor of treatment dropout. $^{24,25}$

\section{Data Analysis}

Data were analyzed using SPSS version 17.0. Besides the descriptive statistics (mean \pm standard deviation, range) for all, the participants were grouped as relapsed $(n=18)$ or non-relapsed $(n=42)$. After checking for normality of the distribution, a between-group comparison was made using the student's two-sampled $t$-test (two-tailed) on the ASI scores for each subscale, as well as each polysomnographic parameter (with $\mathrm{p}<0.05$ ). No statistical analyses were done for the data from the sleep diary and the VAS scores for daytime sleepiness.

\section{Results}

The participants aged 19 to 50 years with a mean age of $35.8 \pm 7.3$ years. A total of $12(20 \%)$ participants relapsed by the end of 3 months, which increased to 18 $(30 \%)$ as seen in the 6 months follow-up (Figure 1). The average total Addiction Score Index (ASI) score for 60 participants was $3.21 \pm 0.22 \mathrm{~L}$, with composite scores (ASI) of $1.3 \pm 0.06,3.48 \pm 0.38,5.45 \pm 0.63,1.2 \pm 0.19$, $3.93 \pm 0.9$, and $2.61 \pm 0.8$ for medical status, employment/support status, alcohol/drugs status, legal status, family/social relationships, and psychiatric status, respectively.

As evident from the sleep diary records of the participants, initial insomnia decreased progressively over 6 months following detoxification for all participants (Figure 2). The VAS scores for self-reported daytime 


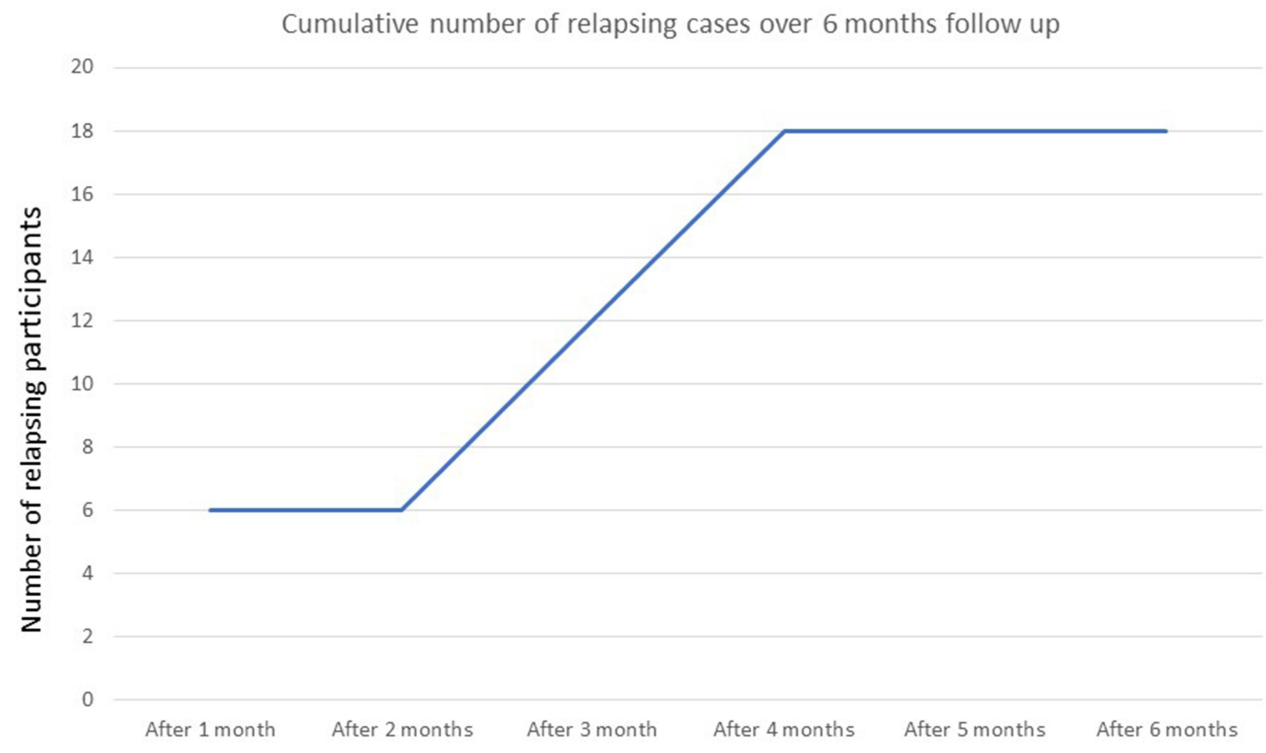

- Initial Insomnia

Figure I Cumulative relapse cases over six months of follow-up. The $x$-axis on the graph represents the time (in months) and $y$-axis depicts the number of cases. $10 \%$, $20 \%$, and $30 \%$ of patients relapsing by the second, third, and fourth months consecutively, plateauing afterward.

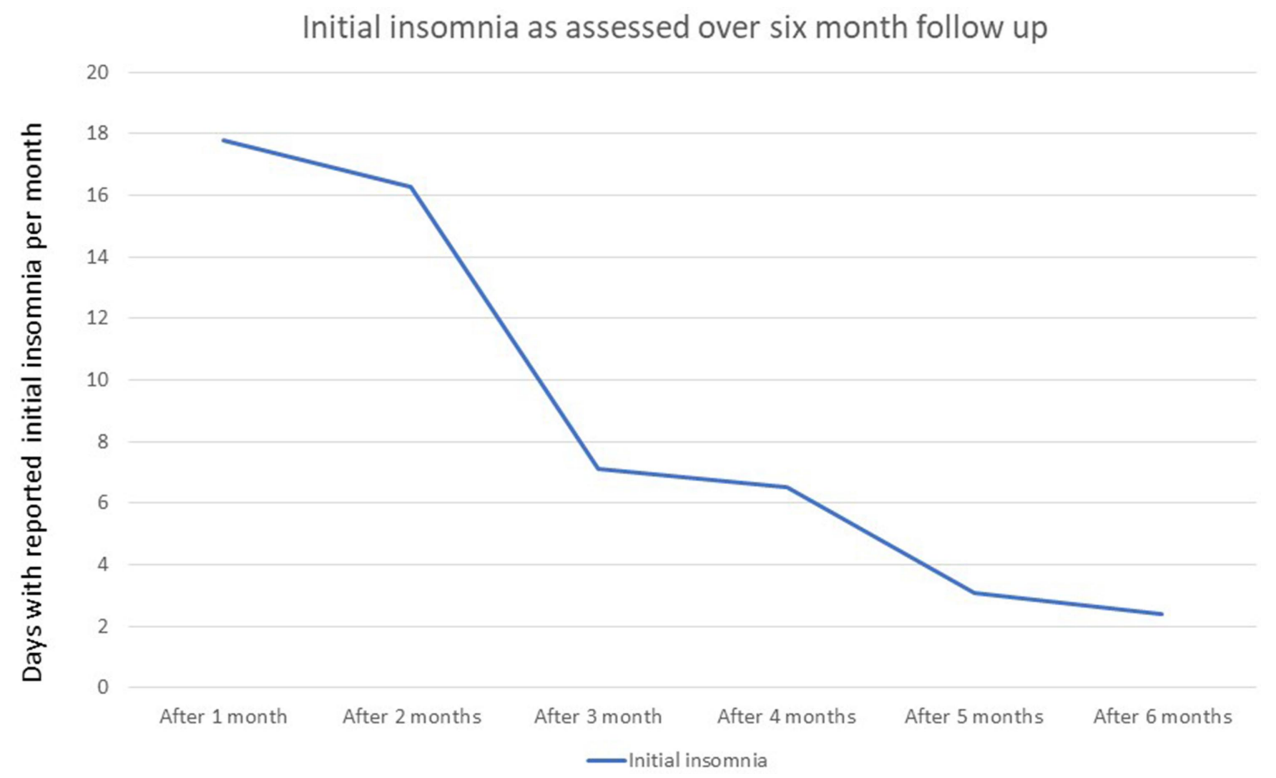

Figure 2 Initial insomnia was subjectively measured over a six-month follow-up period. The graph depicts the number of days in a month (y-axis) for which the subject experienced initial insomnia during sleep during the six months of follow-up (x-axis). Initial insomnia decreased progressively over six months which shed light on the importance of assessing initial insomnia as part of protracted abstinence syndrome.

sleepiness also decreased over this period indicating an improvement in the sleep architecture following detoxification (Figure 3). These results are suggestive of the importance of assessing sleep subjectively for all detoxified patients in the PA phase.

\section{ASI Subscale Scores: Group-Wise Comparison}

The mean \pm SD and range values along with group-wise comparison data are presented in Table 1. On comparing the means of each subscale score for both the groups, only 


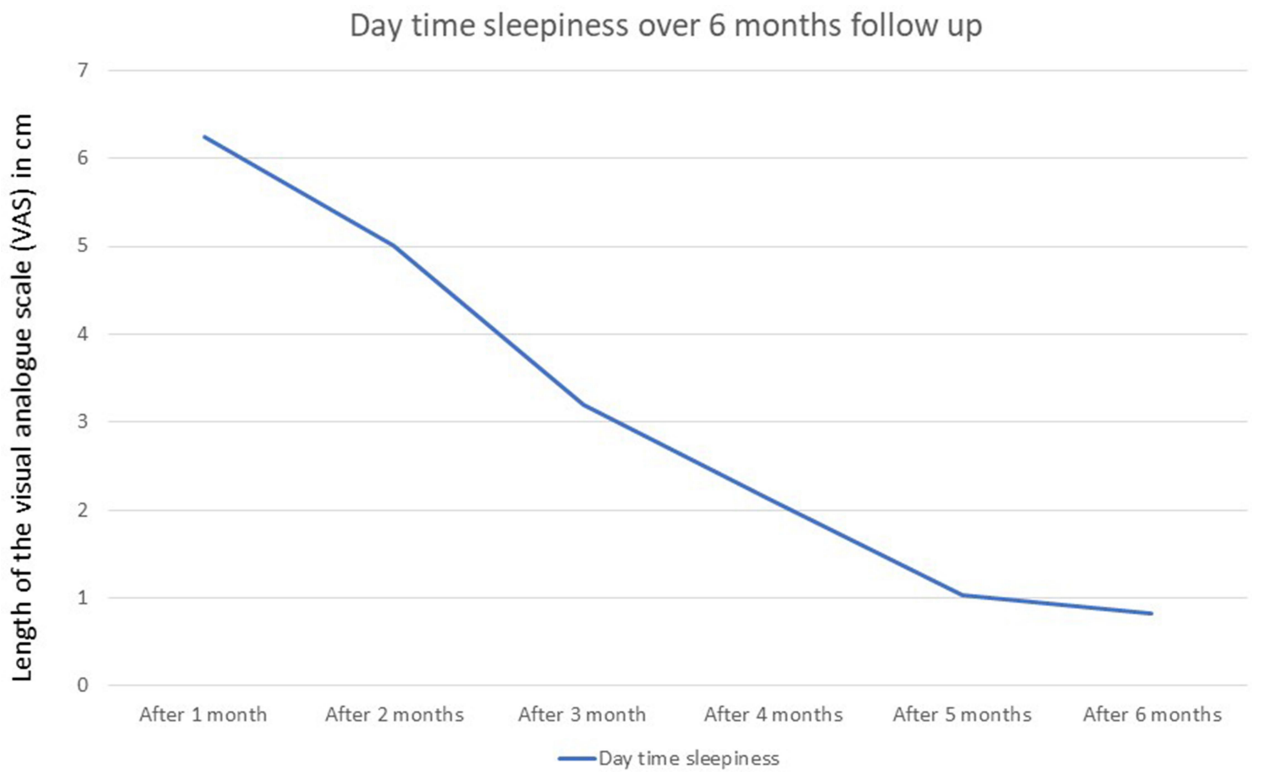

Figure 3 Daytime sleepiness was subjectively measured by the visual analog scale over six months follow-up period. The $x$-axis on the graph represents the time (in months) and $y$-axis depicts the length of VAS (in $\mathrm{cm}$ ) as marked by the patient daily. Daytime sleepiness decreased progressively over 6 months highlighting the importance of assessing it as part of the protracted abstinence syndrome.

the ASI legal problems score showed statistically significant differences between the two groups $(\mathrm{t}=16.537$, $\mathrm{p}=0.001$ ). All other subscales were not statistically different for the two groups of participants.

\section{Polysomnographic Parameters: Group-Wise Comparison}

Eleven parameters were compared for both the groups. Table 2 contains the descriptive, as well as the $t$-test results for the group-wise comparison for polysomnographic parameters. Four out of the 11 parameters showed statistically significant differences between the relapsing and non-relapsing participants, namely percentage of NREM stage I $(\mathrm{t}=22.792, \mathrm{p}=0.001)$, percentage of NREM stage II $(\mathrm{t}=10.285, \mathrm{p}=0.002), \quad$ limb movement index (LMI) $(\mathrm{t}=7.474, \mathrm{p}=0.009)$, and arousal index $(\mathrm{t}=2.65, \mathrm{p}=0.001)$.

\section{Discussion}

The study aimed to assess sleep and sleep structure psychometrically and via polysomnography in detoxified heroin use patients over 6 months. With $30 \%$ of participants relapsing by the end of 6 months, only the ASI legal problems subscale differed between the relapsing and abstinent participants. Whereas on polysomnography, the percentage of NREM stages I and II, limb movement, and arousal indices were different for the two groups. The subjective self-reported sleep characters improved for all participants in the follow-up period.

In the present study, only the legal subscale of ASI was different between the two groups. Previous studies, however, suggest ASI be among the significant predictors of relapse. $^{26,27}$ This difference may be explained by the fact that the participants recruited for the present study had a different profile than the previous studies which included poly-drug users and opiate-misuse patients detoxified and maintained on substitution therapy (not authorized in the health system at the place of conduct of the present research). Mono drug users and detoxification with an alpha-2 agonist-assisted protocols that are still widely in use in our health service system may help reveal rather sample-specific sensitive differences. A poly-drug use profile also points to the extent of behavioral change that the user has in his/her lifestyle, which presents as a multitude of personal and social problems, that can be measured suitably on the ASI, and hence higher scores on other subscales as well.

It is well documented in the literature that both insomnia and excessive sleepiness are risk factors for relapse associated with many drugs that are misused including opiates, amphetamine, cocaine, and alcohol. ${ }^{28}$ Moreover, the presence of insomnia and daytime sleepiness even in the absence of drug-misuse poses a high risk for relapse after detoxification. ${ }^{28-31}$ Our results are in agreement with 
Table I Addiction Severity Index (ASI) Subscales for Both Relapsed $(n=18)$ and Non-Relapsed Groups ( $n=42)$. Only the Legal Problems Subscale of the ASI Was Significantly Higher Among Relapsing Compared to Non-Relapsing Patients

\begin{tabular}{|c|c|c|c|c|}
\hline & Relapsed $(n=18)$ & No Relapsed $(n=42)$ & t Statistic & $P(<0.05) *$ \\
\hline $\begin{array}{l}\text { ASI-Medical Problems } \\
\text { Range } \\
\text { Mean } \pm \text { S.D. }\end{array}$ & $\begin{array}{l}1.31-1.56 \\
1.43 \pm 0.09\end{array}$ & $\begin{array}{l}1.22-1.56 \\
1.38 \pm 0.11\end{array}$ & 1.65 & 0.086 \\
\hline $\begin{array}{l}\text { ASI-Employment/Financial } \\
\text { Range } \\
\text { Mean } \pm \text { S.D. }\end{array}$ & $\begin{array}{l}2.96-3.99 \\
3.45 \pm 0.37\end{array}$ & $\begin{array}{l}2.89-4.22 \\
3.59 \pm 0.36\end{array}$ & 1.07 & 0.156 \\
\hline $\begin{array}{l}\text { ASI-Drug Problems } \\
\text { Range } \\
\text { Mean } \pm \text { S.D. }\end{array}$ & $\begin{array}{l}4.1-5.74 \\
4.94 \pm 0.50\end{array}$ & $\begin{array}{l}4.12-6.33 \\
5.23 \pm 0.69\end{array}$ & 1.69 & 0.106 \\
\hline $\begin{array}{l}\text { ASI-Alcohol Problems } \\
\text { Range } \\
\text { Mean } \pm \text { S.D. }\end{array}$ & $\begin{array}{l}4.2-5.3 \\
4.77 \pm 0.68\end{array}$ & $\begin{array}{l}3.9-6.5 \\
4.52 \pm 0.82\end{array}$ & 1.25 & 0.211 \\
\hline $\begin{array}{l}\text { ASI-Legal Problems } \\
\text { Range } \\
\text { Mean } \pm \text { S.D. }\end{array}$ & $\begin{array}{l}0.9-1.47 \\
1.13 \pm 0.20\end{array}$ & $\begin{array}{l}0.99-1.63 \\
1.33 \pm 0.16\end{array}$ & 16.53 & $0.001 *$ \\
\hline $\begin{array}{l}\text { ASI-Social Problems } \\
\text { Range } \\
\text { Mean } \pm \text { S.D. }\end{array}$ & $\begin{array}{l}2.6-5.45 \\
3.88 \pm 0.99\end{array}$ & $\begin{array}{l}2.69-5.62 \\
4.34 \pm 0.94\end{array}$ & 1.99 & 0.089 \\
\hline $\begin{array}{l}\text { ASI-Psychological Problems } \\
\text { Range } \\
\text { Mean士S.D. }\end{array}$ & $\begin{array}{l}1.47-4.15 \\
2.43 \pm 0.87\end{array}$ & $\begin{array}{l}1.49-3.9 \\
2.83 \pm 0.70\end{array}$ & 3.58 & 0.064 \\
\hline
\end{tabular}

Note: ${ }^{*} p<0.05$.

these findings in terms of the presence of insomnia and excessive daytime sleepiness during the 6 months following detoxification, particularly high during the first 3 months which coincide with a higher relapse rate. This pattern can be easily observed by putting together the three figures, indicating a tendency to fall back to drug use to counter the state of lethargy and low arousal.

Polysomnography gives an objective measure of how much the sleep architecture has been affected, and whether it falls within the pathological limits to require treatment. As discussed, the stress, sleep, and opioid systems are closely chemically linked, opioid intake may help the user counter the effects of poor sleep and cope with the associated stress. Hence, the present study aimed to evaluate whether the polysomnographic parameters can serve as predictors for relapse in detoxified patients facing sleep disturbances. In this study for polysomnographic parameters, the percentage of NREM stages I\&II as well as limb movement and arousal indices came out to be significantly different between both relapsing and non- relapsing groups. Our findings come in agreement with the previous authors. ${ }^{32}$ Consequently, these can potentially be assessed on a larger scale as early risk factors for relapse. A previous study by Howe et al that was conducted at about 5-7 days of acute abstinence after chronic heroin use reported a reduced total sleep time, REM stage II sleep, and slow-wave sleep. Other studies have also described an increased interval for sleep onset, more wake time after the onset of sleep, and REM latency as compared to healthy sleepers. ${ }^{33}$ Also, it has been reported that during the first 3 weeks of abstinence, a decreased sleep efficiency, reduced total sleep time, increased arousal on arousal index, a prolonged sleep latency, increased NREM stage 1 and 2, and a decreased slow-wave sleep (SWS) were prominent in detoxified subjects compared to healthy control. ${ }^{19,34}$ In contrast, our results did not point to any differences in the SWS. A possible explanation could be the small sample size of our study, and the participants were being subjected to alpha-2 agonist-assisted protocols rather than substitution assisted detoxification. 
Table 2 Polysomnographic Parameters Scored One Week After Detoxification in Relapsing and Non-Relapsing Patients. Percentage of NREM Stages I and 2, Limb Movement Index and Arousal Index Were Statistically Different Between the Relapsed and NonRelapsed Groups

\begin{tabular}{|c|c|c|c|c|}
\hline & Relapsed $(n=18)$ & No Relapsed $(n=42)$ & $t$ Statistic & $\mathrm{P}(<0.05)^{*}$ \\
\hline TST Total Sleep Time & & & 0.10 & 0.753 \\
\hline Range & $|82.5-36| .5$ & | 28.5-349.5 & & \\
\hline Mean \pm S.D. & $276.1 \pm 89.3$ & $266.9 \pm 75.1$ & & \\
\hline Sleep Efficiency & & & 1.09 & 0.301 \\
\hline Range & $9 \mid-6.3$ & $85.7-99.5$ & & \\
\hline Mean \pm S.D. & $95.0 \pm 2.3$ & $93.8 \pm 3.2$ & & \\
\hline Stage I NREM (\%) & & & 22.79 & $0.001 *$ \\
\hline Range & $|-| \mid$ & $0-7$ & & \\
\hline Mean \pm S.D. & $6.1 \pm 4.7$ & $1.6 \pm 1.7$ & & \\
\hline Stage 2 NREM (\%) & & & 10.28 & $0.002 *$ \\
\hline Range & $45-60.3$ & $45-78.6$ & & \\
\hline Mean $\pm S D$. & $53.5 \pm 6.8$ & $64.2 \pm 9.4$ & & \\
\hline Stage 3 NREM (\%) & & & 2.3 & 0.136 \\
\hline Range & $11.2-43$ & $0-43$ & & \\
\hline Mean \pm S.D. & $25.9 \pm 14.3$ & $18.6 \pm 12.6$ & & \\
\hline REM (\%) & & & 0.88 & 0.358 \\
\hline Range & $11-13$ & $0-18$ & & \\
\hline Mean \pm S.D. & $12.2 \pm 1.1$ & $10.0 \pm 5.3$ & & \\
\hline LMI Limb Movement Index & & & 7.47 & $0.009 *$ \\
\hline Range & $3-99.9$ & $0.7-52.4$ & & \\
\hline Mean \pm S.D. & $5.9 \pm 2.5$ & $19.9 \pm 15.2$ & & \\
\hline Sleep Latency & & & 0.35 & 0.557 \\
\hline Range & $2.5-16.5$ & $0-25$ & & \\
\hline Mean \pm S.D. & $8.4 \pm 6.5$ & $6.7 \pm 8.2$ & & \\
\hline REM Latency & & & 1.71 & 0.198 \\
\hline Range & $57.5-205$ & $0-2 \mid 4.5$ & & \\
\hline Mean \pm S.D. & $109.9 \pm 60.4$ & $77.3 \pm 68.8$ & & \\
\hline Arousal Index & & & 2.65 & $0.001 *$ \\
\hline Range & $15.0-24.2$ & $16.0-24.1$ & & \\
\hline Mean \pm S.D. & $19.2 \pm 3.22$ & $17 \pm 2.65$ & & \\
\hline Respiratory Disturbance Index & & & 1.65 & 0.110 \\
\hline Range & $0.0-6.0$ & $0.0-5.0$ & & \\
\hline Mean \pm S.D. & $3.00 \pm 1.03$ & $2.5 \pm 0.98$ & & \\
\hline
\end{tabular}

Note: $*_{p}<0.05$.

The association between disturbance in sleep patterns and the tendency to relapse to drug-use remains unmapped and hence requires a deeper analysis. There is evidence of a relation between dyssomnia (an umbrella term used to describe sleep disorders characterized by the inability to fall or maintain sleep) and the rate of abstinence in patients with a history of drug use, who are on substitution therapy using methadone and buprenorphine. ${ }^{35,36} \mathrm{~A}$ recent review based on 3 longitudinal studies and 15 cross-sectional studies, using diverse methodological assessment of sleep disturbances as well as varying durations, confirms the influence of sleep deprivation and dyssomnia on relapsetendencies in drug-use patients on substitution therapy. ${ }^{32}$ Dyssomnia is a broad symptom that can be assessed 
subjectively or objectively using various psychometric tests, and also electrophysiological examination using polysomnography. ${ }^{37,38}$ Thus, further research is warranted to assess sleep parameters deeply and set cut-off scores that may be used as prognosticators for the risk of relapse among other mental disorders.

\section{Limitations}

To extrapolate and generalize results for the whole adult Egyptian population, a larger sample should be recruited to reduce the effect of type II statistical error, and consecutively increase study power. However, the sample in this study was sufficient to yield statistically significant results. Secondly, only the male gender was considered for this study, further reducing the scope of application of the results. Further studies should focus on the influence of gender-selective measurement bias and study gendersensitive issues related to addiction. Also, recruiting subjects with existing co-morbidities was avoided to minimize the effect of additional drug use. However, debilitating medical diseases that may influence the subject's sleep and ability to stay abstinent should be considered in future studies. We conducted polysomnography only once; however, the process could have been repeated monthly to allow a thorough assessment of polysomnographic parameters before relapse. Mono-drug users, ie, only heroindetoxified patients who were undergoing an alpha-2 adrenergic agonist assisted detoxification regimen, were recruited. While our health system allows for the aforementioned treatment, future studies should analyze the role of different treatment regimens on sleep structure postdetoxification to expand our knowledge in this respect. Application of more detailed psychometric assessment scales and sleep questionnaires can further enrich future studies as more sleep-related parameters are used in analysis and comparisons.

Though reconsidering potential confounders in a multivariance statistical model for analysis could potentially enrich results and decrease the probability of type II statistical errors related to confusion bias, our sample size did not allow this as it may lead to consecutive multiple subcategorization generating subsequent difficulties and weakness in applying statistical tests on the small-sized subcategorical groups.

\section{Conclusion}

The study aimed to identify the various sleep-related abnormalities in detoxified heroin-misuse patients, and their influence on the user forcing him to backslide, in a six-month follow-up. Consequently, an objective measurement of these aberrations by polysomnography may potentially help predict relapses. It can be concluded from this study that heroin misusers in their protracted abstinence phase face insomnia and excessive daytime sleepiness, which makes them susceptible to relapse. This was evident from the high relapse rate and initial insomnia in the initial 3 months of abstinence. Also, the relapse to drug use may pose a significant threat in terms of legal problems, as manifested in the Addiction Severity Index scoring of the participants. As for polysomnography is concerned, the parameters - percentage of NREM stage I and II, limb movement index, and arousal index, can be the potential candidates for predictors of relapse. Monitoring these parameters over a time interval may help predict potential relapses, and devise a multi-disciplinary treatment to overcome them.

\section{Disclosure}

Authors declare that they have no conflicts of interest in this work.

\section{References}

1. The Central Agency for Public Mobilization and Statistics (CAPMAS), Egypt. Egyptian Population Report. 2006.

2. Amin G. What Happened to Egyptians: Evolution of Egyptian Society1945/1995. Cairo: Dar El Shorouk Press; 2007.

3. Mental Health Secretariat, Ministry of Health, Information Department. Monthly and Annual Admission Reports, 2000-2010. Cairo: Mental Health Secretariat; 2011.

4. Rakhawy Y. Ministry of Health: National Research on Addiction: Use, Abuse, Dependency, and Addiction: Preliminary Report. Cairo: Ministry of Health; 1996.

5. National Centre for Social and Criminological Research. The Use of Psychoactive Substances Among Secondary and Technical Secondary School Students. Vol. 10. Cairo: National Centre for Social and Criminological Research; 2003.

6. Ghanem M, Gadallah M, Meky FA, et al. National survey of the prevalence of mental disorders in Egypt: preliminary survey. East Mediterr Health J. 2009;15(1):65-75. doi:10.26719/2009.15.1.65

7. World Health Organization. Guide to Drug Abuse Epidemiology. Geneva: World Health Organization; 2000.

8. Hamdi E, Gawad T, Khoweiled A, et al. Lifetime prevalence of alcohol and substance. Use in Egypt: a community survey. Subst Abus. 2013;34(2):97-104. doi:10.1080/08897077.2012.677752

9. Grant JE, Chamberlain SR. Expanding the definition of addiction: DSM-5 vs. ICD-11. CNS Spectr. 2016;21(4):300-303. doi:10.1017/ S1092852916000183

10. Brower KJ, Perron BE. Sleep disturbance as a universal risk factor for relapse in addictions to psychoactive substances. Med Hypotheses. 2010;74(5):928-933. doi:10.1016/j.mehy.2009.10.020

11. Wilkerson AK, Sahlem GL, Bentzley BS, et al. Insomnia severity during early abstinence is related to substance use treatment completion in adults enrolled in an intensive outpatient program. $J$ Subst Abuse Treat. 2019;104:97-103. doi:10.1016/j.jsat.2019.06.003 
12. Reppert SM, Artman HG, Swaminathan S, et al. Vasopressin exhibits arrhythmic daily pattern in cerebrospinal fluid but not in blood. Science. 1981;213(4513):1256-1257. doi:10.1126/science.7268432

13. Gutstein H, Akil H. Opioid analgesics. In: Hardman JG, Limbird LE, Gilman AG, editors. Goodman and Gilman's the Pharmacological Basis of Therapeutics. 11th ed. New York: McGraw-Hill; 2005.

14. Wilson L, Dorosz L. Possible role of the opioid peptides in sleep. MedHypotheses. 1984;14(3):269-280. doi:10.1016/0306-9877(87) 90128-9

15. Weitzman RE, Fisher DA, Minick S, et al. Beta-endorphin secretion of arginine vasopressin in vivo. Endocrinology. 1977;101 (5):1643-1646. doi:10.1210/endo-101-5-1643

16. Przewlocki R, Lason W, Konecka AM, et al. The opioid peptide dynorphin, circadian rhythms, and starvation. Science. 1983;219 (4580):71-73. doi:10.1126/science.6129699

17. Lord JA, Waterfield AA, Hughes J, et al. Endogenous opioid peptides: multiple agonists and receptors. Nature. 1977;267 (5611):495-499. doi:10.1038/267495a0

18. Robert C, Stinus L, Limoge A. Sleep impairments in rats implanted with morphine pellets. Neuropsychobiology. 1999;40(4):214-217. doi:10.1159/000026622

19. Howe RC, Hegge FW, Phillips JL. Acute heroin abstinence in man: I. Changes in behavior and sleep. Drug Alcohol Depend. 1980;5 (5):341-356. doi:10.1016/0376-8716(80)90160-X

20. Younes M, Kuna ST, Pack AI, et al. Reliability of the American academy of sleep medicine rules for assessing sleep depth in clinical practice. J Clin Sleep Med. 2018;14(2):205-213. doi:10.5664/ jcsm. 6934

21. Weitzman ED, Czeisler CA, Zimmerman JC, et al. Chronobiological disorder: analytic and therapeutic techniques. In: Guilleminault $\mathrm{C}$, ed. Disorders of Sleeping and Waking: Indications and Techniques. California: Addison-Wesley; 1982:297-329.

22. Folstein MF, Luria R. Reliability, validity, and clinical application of the visual analog mood scale. Psychol Med. 1973;3(4):479-486. doi:10.1017/S0033291700054283

23. Rechtschaffen A, Kales A, Eds., A Manual of Standardized Terminology, Techniques, and Scoring System for Sleep Stages of Human Subjects. Bethesda MD: Publication NIH; 1968:204.

24. Alterman AI, Kampman K, Boardman CR, et al. A cocaine-positive baseline urine predicts outpatient treatment attrition and failure to attain initial abstinence. Drug Alcohol Depend. 1997;46(1-2):79-85. doi:10.1016/S0376-8716(97)00049-5

25. Shah S, De Matteo D, Keesler M, et al. Addiction severity index scores and urine drug screen sat baseline as predictors of graduation from drug court. Crime Delinq. 2015;61(9):1257-1277. doi:10.1177/ 0011128713496007

26. Chen T, Zhong N, Du J, et al. Polydrug use patterns and their impact on relapse among heroin-dependent patients in Shanghai, China. Addiction. 2019;114(2):259-267. doi:10.1111/add.14451
27. K8rupitsky E, Zvartau E, Blokhina E, et al. Anhedonia, depression, anxiety, and craving in opiate-dependent patients stabilized on oral naltrexone or an extended-release naltrexone implant. $\mathrm{Am}$ J Drug Alcohol Abuse. 2016;42(5):614-620. doi:10.1080/ 00952990.2016 .1197231

28. Vetrova MV, Kuchmenko DN, Genina IN, et al. The prevalence of the sleep disturbances among the patients with substance use disorders. Bull Sib Med. 2020;18(4):26-35. doi:10.20538/1682-0363-20194-26-35

29. Geoffroy PA, Tebeka S, Blanco C, et al. Shorter and longer durations of sleep are associated with an increased twelve-month prevalence of psychiatric and substance use disorders: findings from a nationally representative survey of US adults (NESARC-III). J Psychiatr Res. 2020;124:34-41. doi:10.1016/j.jpsychires.2020.02.018

30. Neale J, Vitoratou S, Lennon P, et al. Development and early validation of a patient-reported outcome measure to assess sleep amongst people experiencing problems with alcohol or other drugs. Sleep. 2018;41(4):41. doi:10.1093/sleep/zsy013

31. Wojciechowski TW. Heterogeneity in the development of drug use versatility: risk factors for polydrug use throughout the life-course. Subst Use Misuse. 2019;54(5):758-768. doi:10.1080/ 10826084.2018.1536721

32. Tripathi R, Rao R, Dhawan A, et al. Opioids, and sleep - a review of the literature. Sleep Med. 2020;67:269-275. doi:10.1016/j. sleep.2019.06.012

33. Gowing L, Ali R, White JM. Buprenorphine for the management of opioid withdrawal. Cochrane Database Syst Rev. 2009;(3): CD002025.

34. Asaad T, Ghanem M, Abdel Samee A, et al. Sleep profile in patients with chronic opioid abuse: a polysomnographic evaluation in an Egyptian sample. Addict Disord Their Treat. 2011;10(1):21-28. doi:10.1097/ADT.0b013e3181fb2847

35. Kelly E, Finan PH, Andrew Tompkins D, Strain EC. Frequency and correlates of sleep disturbance in methadone and buprenorphine-maintained patients. Addict Behav. 2018;76:8-14. doi:10.1016/j.addbeh.2017.07.016

36. Vetrova M, Skurat E, Rybakova K, et al. The longitudinal analysis of sleep quality and relapse: survey data from the cohort of people with substance use disorders. Eur Neuropsychopharmacol. 2020;40:S354. doi:10.1016/j.euroneuro.2020.09.458

37. Chung K-F. Subjective-objective sleep discrepancy in schizophrenia, behavioral. Sleep Med. 2020;18(5):653-667. doi:10.1080/ 15402002.2019.1656077

38. Kurtis Mónica M, Roberta B, Carmen R-B, João FM, Pablo M-M. A review of scales to evaluate sleep disturbances in movement disorders. Front Neurol. 2018;9:369. doi:10.3389/fneur.2018.00369
Neuropsychiatric Disease and Treatment

\section{Publish your work in this journal}

Neuropsychiatric Disease and Treatment is an international, peerreviewed journal of clinical therapeutics and pharmacology focusing on concise rapid reporting of clinical or pre-clinical studies on a range of neuropsychiatric and neurological disorders. This journal is indexed on PubMed Central, the 'PsycINFO' database and CAS, and is the official journal of The International Neuropsychiatric Association (INA). The manuscript management system is completely online and includes a very quick and fair peer-review system, which is all easy to use. Visit http://www.dovepress.com/testimonials.php to read real quotes from published authors. 